\title{
EDITORIAL
}

\section{Tecnología e innovaciones de papa como puente crítico para responder a los desafíos de seguridad alimentaria y promover los agronegocios en América Latina}

\author{
A. Devaux ${ }^{1 / *}$
}

Recibido: $22 / 06 / 2018$

Aceptado: $30 / 06 / 2018$

Accesible en línea: June 2018

Muchos científicos latinoamericanos dedicados al estudio de la papa habían esperado durante largo tiempo este momento. Del 27 al 31 de mayo, el X Congreso Mundial de la Papa (CMP) y el XXVIII Congreso de la Asociación Latinoamericana de la Papa (ALAP) se llevaron a cabo juntos por primera vez, en la histórica ciudad de Cusco, Perú. El Congreso Mundial de la Papa se realiza cada tres años, y después de haber tenido como sede América del Norte, Europa, Sudáfrica, China y Nueva Zelanda, el momento largamente esperado de un congreso en América del Sur, la cuna de la papa, finalmente llegó.

Este año, el evento reunió a más de 800 científicos, representantes de la industria de la papa y otros expertos en Cusco. Al mismo tiempo, el XXVIII Congreso ALAP, que tiene lugar cada dos años, reunió a estudiantes, investigadores, empresarios, productores y otros actores en la cadena de valor. La agenda de los congresos combinados incluyó presentaciones plenarias, sesiones técnicas, talleres, intercambios de información, descubrimientos e innovaciones útiles, todos presentados en el marco de tres temas principales: biodiversidad, seguridad alimentaria y negocios. Los siete temas técnicos elegidos para este evento incluyeron: • Cambio Climático y Sistemas Agroalimentarios de Papa. - Tendencias en el Consumo y Mercados de Papa. - Desarrollo de Variedades de Papa y Biotecnología - Plagas y Enfermedades de la Papa - Manejo del Cultivo de Papa - Tecnología de Poscosecha y Procesamiento - Biodiversidad de la Papa y su Relación con el Mejoramiento, Nutrición y Salud. La mayoría de las 164 presentaciones provenían de América Latina, que representaba el $68 \%$ del total, seguida por Europa, con el 19\%, África, con el 7\%, el 5\% de Asia y el 1\% de Oceanía (World Potato Congress, 2018).

Este Congreso fue una excelente oportunidad para mostrar investigaciones sobre el uso sostenible de la biodiversidad de la papa, centrándose en su relación con la salud y nutrición; su contribución a los ingresos de los agricultores; y su potencial para responder a la demanda mundial de alimentos que crecerá en un 50\% para el año 2030. Las diferentes sesiones también fueron una oportunidad para debatir sobre la seguridad alimentaria abordando los problemas del desarrollo varietal, la biotecnología, el control de plagas y enfermedades, el manejo de cultivos y de la semilla y el cambio climático. Además, el consumo y los mercados, las tecnologías de poscosecha y procesamiento y las innovaciones culinarias fueron de especial interés para las personas involucradas en el negocio de la papa.

Las actividades del CMP y el congreso ALAP fueron complementadas por varios eventos paralelos. El día anterior al congreso, científicos de todo el continente americano se juntaron para una reunión de la Red Latinoamericana para la Investigación del Tizón Tardío, conocida

\footnotetext{
* Contacto del autor: E-mail: A.DEVAUX@cgiar.org

1 Director Regional de América Latina y el Caribe (LAC) del CIP.
} 
como la Red Tizón Latino. Esta red se fundó para coordinar la investigación sobre esta enfermedad a nivel regional, y se inspiró en la red europea de investigación EuroBlight. Vinculado a esta reunión, el CIP y la Asociación Europea de Investigación de la Papa (EAPR) organizaron un taller con socios públicos y privados en los Andes para promover la colaboración en investigación y desarrollo (I\&D) para el impulso de soluciones biológicas para controlar plagas y enfermedades de la papa y mejorar la productividad de la papa en una manera sostenible en los Andes. El CMP y el CIP colaboraron en la organización de un evento para lanzar la campaña Imagina un mundo sin papas", en donde representantes de compañías multinacionales, asociaciones de la papa, entidades del sector público, centros de investigación y organizaciones internacionales fueron invitados a unirse la campaña y usar su mensaje principal para promover la papa. El objetivo de esta campaña es garantizar que el mundo comprenda la importancia y el potencial de este tubérculo para brindar soluciones a los desafíos mundiales actuales de alimentación y nutrición, tal como lo ha hecho desde que se expandió por primera vez alrededor del mundo desde los Andes hace más de 500 años. Las presentaciones, sesiones de posters y talleres del CMP 2018 y el Congreso ALAP se complementaron con un día de campo en el que los participantes tuvieron la oportunidad de unirse a uno de los dos viajes organizados a sitios conocidos por su conservación e investigación de papa: El Parque de la Papa y la Estación Experimental Andenes del Instituto Nacional para la Innovación Agrícola (INIA).

El CMP y el Congreso ALAP fueron una buena oportunidad para presentar resultados de investigación e innovación en papa en América Latina y el Caribe y analizar de cerca el papel de la región en la producción mundial de papa. El suministro promedio anual de papa en la región de LAC ha aumentado de 7.2 millones de toneladas en el período 1961-1963 a 19.6 millones de toneladas en 2011-2013. A modo de comparación, las tasas de crecimiento en la producción de papa en Asia y África promediaron más del 4\% para un período similar, es decir, más del doble que en LAC. La mayor parte de esa producción está orientada al consumo humano ( $74 \%$, manteniendo esta tendencia durante todo el período) y, según la FAO, representa un nivel todavía relativamente bajo de procesamiento con fines industriales del 1\% (FAOSTAT 2017).

El papel que desempeña la papa en las dietas en LAC varía de un área a otra: de alimento básico para productores/consumidores en zonas alto-andinas, a un vegetal complementario para hogares urbanos en la mayor parte de Sudamérica, a un vegetal complementario relativamente caro en gran parte de América Central y el Caribe, y finalmente como un producto popular de comida rápida en forma de papas fritas en los mercados urbanos de toda la región (Scott, 2011). El consumo per cápita de papa en América Latina aumentó ligeramente de $22 \mathrm{~kg} /$ persona en promedio entre 1961 y 1963 a $25 \mathrm{~kg} /$ persona entre 2011 y 2013. Pero estas cifras regionales no reflejan las diferencias importantes en las tendencias a nivel subregional y nacional. Perú, es uno de los países donde el consumo de papa ha crecido significativamente, llegando a $85 \mathrm{~kg} /$ persona en 2015. Esto se debe a diversas políticas público-privadas, infraestructura rural, expansión del comercio de supermercados centrado en la papa y promoción por parte del sector gastronómico de platos andinos incluyendo la papa y sus productos. Brasil y México han aumentado su consumo, aunque sus valores absolutos siguen siendo bajos. Sin embargo, se prevé que el mayor aumento en la producción y el consumo de papas en América del Sur en las próximas décadas ocurra en Brasil. En el país más poblado de la región, el consumo actual de papa per cápita sigue siendo relativamente modesto en comparación con otros países. Mientras tanto, Argentina y Colombia experimentan tendencias a la baja en el consumo de papa (Scott \& Kleinwechter 2017). 
Muchos agricultores en LAC, pero especialmente en América del Sur, están interesados en el cultivo de papa debido a su adaptabilidad y período de madurez relativamente corto, y al hecho de que puede ser tanto un cultivo de seguridad alimentaria como un generador de ingresos. Además de ser una excelente fuente de carbohidratos, las papas tienen niveles relativamente altos de vitamina $\mathrm{C}$, hierro, zinc y antioxidantes, lo que significa que pueden contribuir a mejorar la nutrición, así como la seguridad alimentaria.

Las condiciones de cultivo varían de un país a otro y de una región a otra. Algunas áreas tienen temporadas cortas de cultivo, mientras que otras tienen dos estaciones, y el riesgo de sequía o demasiada precipitación está creciendo debido al cambio climático. Los agricultores en muchas áreas de LAC también deben lidiar con la presión de enfermedades y plagas. Estos y otros factores son responsables de las grandes diferencias en los rendimientos de papa entre los países de la región. Si bien los países de la región de los Andes tienen rendimientos promedio inferiores a las 20 toneladas de papas por hectárea, la mayoría de los agricultores del Cono Sur producen más de 40 toneladas por hectárea. Cerrar esa brecha de rendimiento es esencial para aprovechar todo el potencial de la papa para mejorar la seguridad alimentaria y los ingresos de las familias de pequeños agricultores, y es un objetivo central de las organizaciones de investigación para el desarrollo como el CIP y las instituciones nacionales de investigación y desarrollo agrícola.

Durante el Congreso, se describieron algunas de las áreas más importantes en las que los centros de investigación agrícola y sus socios están trabajando para ayudar a los agricultores a cerrar la brecha de rendimiento.

El mejoramiento genético ha sido durante mucho tiempo una prioridad para las organizaciones dedicadas a ayudar a los productores de papa en el mundo en desarrollo a aumentar sus rendimientos. Con los años, los investigadores han desarrollado variedades de alto rendimiento con resistencia al tizón tardío y las enfermedades virales, que son probablemente las principales limitaciones para los productores de papa de pequeña escala en los trópicos y sub-trópicos. La mayoría de esos agricultores carecen de acceso a semillas libres de enfermedad, y no tienen los recursos para comprar los fungicidas necesarios para controlar el patógeno del tizón tardío. También es vital que los programas de mejoramiento de la papa se centren en tecnologías climáticamente inteligentes, teniendo en cuenta las amenazas del cambio climático y su posible impacto en los agricultores, especialmente en los Andes, América Central y el Caribe. Por lo tanto, se necesitan variedades resistentes, precoces $\mathrm{y}$ adaptadas que puedan producir suficiente alimento en condiciones climáticas extremas. También existe una necesidad crucial de desarrollar variedades con mejores características de procesamiento y almacenamiento para responder a los requerimientos de los agronegocios.

Mejorando la calidad, producción y distribución de semillas. Los sistemas de semillas son vitales para el despliegue de variedades mejoradas de papa, pero es importante reconocer que muchos agricultores carecen de acceso a ellos. Esos agricultores seleccionan de su propia cosecha la semilla para la próxima temporada de siembra o la compran de fuentes informales, como los mercados locales, lo que generalmente significa que es de baja calidad sanitaria. Esta es una causa importante de bajos rendimientos, lo que significa menos alimentos y menos dinero para las familias de escasos recursos. Los científicos están recomendando un enfoque integrado que combine sistemas de semillas limpios con multiplicadores de semillas descentralizados y la promoción de capacitación en prácticas de selección en campo y almacenamiento que puedan ayudar a los pequeños agricultores a mejorar la calidad de la semilla de papa que siembran. 
Manejo de cultivos de papa. La investigación ha realizado importantes inversiones para desarrollar, probar y difundir tecnologías y enfoques para ayudar a los pequeños agricultores, hombres y mujeres, a mejorar el manejo de sus cultivos y, de ese modo, aumentar la producción de alimentos. Estas tecnologías incluyen herramientas de apoyo a la decisión para mejorar el control de plagas y enfermedades, métodos de riego que ahorran agua, un mejor manejo del suelo y otros enfoques de agricultura sostenible que pueden permitir a los agricultores mejorar sus rendimientos de papa y su resiliencia frente al cambio climático. La agricultura inteligente es una nueva vía para la optimización del uso de recursos basada en nuevas herramientas de monitoreo y soporte de decisiones. Las herramientas de teledetección y del sistema de información geográfica (SIG) junto con los sistemas de apoyo a las decisiones y las tecnologías agrícolas de precisión pueden contribuir a aumentar la productividad. La investigación para desarrollar el control biológico es popular y se considera que crecerá sustancialmente en la próxima década, pero todavía hay pocos éxitos confirmados en el campo, y aún faltan herramientas de manejo específicas (Velivelli et al., 2014).

Integrando la seguridad alimentaria y el desarrollo de la cadena de valor. Los agronegocios son un sector diverso y complejo y una cadena de valor exitosa requiere que todo el sistema funcione. Existen muchos desafíos para el desarrollo de cadenas de valor de la papa y pueden variar entre países e incluso dentro de los países. El CIP ha trabajado con una serie de socios en iniciativas que han ayudado a los pequeños productores a ir más allá de la agricultura de subsistencia y obtener acceso a mercados de alto valor. Un ejemplo de esto es la colaboración del CIP con los sectores público y privado en la región de los Andes para desarrollar nuevas cadenas de valor para las papas nativas.

Manejo poscosecha. Otra pieza importante del rompecabezas es mejorar el manejo poscosecha, para reducir la pérdida de papas y de la calidad de la papa. Si bien existe una creciente conciencia del desperdicio de alimentos en los niveles del minorista y del consumidor, que es extremadamente importante, debemos prestar más atención a la pérdida poscosecha en el campo, en el transporte y en el almacenamiento. Por lo tanto, las intervenciones para facilitar cadenas de mercado inclusivas deben complementarse con esfuerzos para ayudar a los agricultores a mejorar su manejo y almacenamiento poscosecha. Un estudio reciente de CIP sobre cadenas de valor de papa en Perú y Ecuador documentó pérdidas de entre $12 \%$ y $30 \%$, lo que ilustra la importancia de esta área de investigación.

Todos los que trabajamos para el desarrollo del sector agrícola en esta región necesitamos promover tecnologías y enfoques que puedan ayudar a los agricultores a adaptarse al cambio climático, aumentar su producción y limitar sus impactos ambientales. Estos incluyen variedades de papa resilientes; innovaciones para acelerar la producción de semillas; tecnologías apropiadas para mejorar la detección y el control de las enfermedades y plagas de la papa; y las innovaciones de la cadena de mercado para crear oportunidades de ingresos. A medida que trabajamos en enfoques más integradores de ciencia y desarrollo para ayudar a los agricultores de nuestra región a adaptarse y prepararse para los desafíos futuros, las organizaciones de investigación agrícola deben colaborar con nuevos socios en los sectores gubernamental, empresarial y sin fines de lucro. Al trabajar juntos, y con una mayor variedad de socios, podemos hacer frente a los desafíos y aprovechar todo el potencial de la papa para fortalecer la seguridad alimentaria, reducir la pobreza y permitir que los agricultores de la región tengan mejores medios de vida.

Me gustaría concluir esta editorial con una nota que honra la memoria del Dr. Patricio Malagamba, quien durante años apoyó la publicación de la revista ALAP, proporcionó orientación en artículos sobre investigación en papa y contribuyó al desarrollo del sector en América Latina. 


\section{Referencias}

FAO. (2017). FAOSTAT Database. http://www.fao.org/faostat/en/\#data/QC (accedido el 24 de noviembre, 2017)

World Potato Congress. (2018). Abstract Book 10th WPC- XXVIII ALAP 2018 Congress: Biodiversity, Food Security and Business. Instituto Nacional de Innovación Agraria-INIA. Cusco, Perú. 168 pp.

Scott G. (2011). Growth Rates for Potatoes in Latin America in Comparative Perspective: 1961-07. Am. J. Pot Res 88:143-152.

Scott, G.J. \& Kleinwechter, U. (2017). Future Scenarios for Potato Demand, Supply and Trade in South America to 2030. Potato Res. 60: 23.

Velivelli, S.L.S., Sessitsch, A. and Prestwich, B.D. (2014). The role of microbial inoculants in integrated crop management systems, Potato Research, 57 (3-4), 291-309. 